Preface

\section{Surgeon Health}

\author{
Jennifer S. Davids, MD, FACS, FASCRS ${ }^{1}$ \\ ${ }^{1}$ Division of Colon and Rectal Surgery, UMass Memorial Medical \\ Center, Worcester, Massachusetts
}

Clin Colon Rectal Surg 2019;32:405-406.

It is more challenging than ever to be a surgeon in the current health care environment. In the wake of declining reimbursements, pressure to increase clinical productivity, electronic medical record requirements, and ergonomically strenuous operative platforms, inevitably surgeons are becoming the "second victim." If we fall prey to these mounting physical and psychological demands, our colleagues, trainees, families, and, ultimately, our patients may suffer.

As surgeons, often we are the last to prioritize our own physical and emotional well-being. The culture of surgery is gradually responding to the growing body of evidence that the wellness of surgeons matters. In fact, the Accreditation Council on Graduate Medical Education now requires physician well-being education of all residents in response to the rising concern, particularly among the millennials, over the alarming rates of substance abuse and physician suicide.

The goal of this volume is to address many important aspects of surgeon health. You will learn how to recognize burnout and build resilience, improve ergonomics, and minimize workplace exposures. We will also discuss strategies for work-life integration and specific considerations for the pregnant surgeon. Lastly, we address how to combat the unique stressors of early career surgeons as well as effectively transitioning from clinical practice

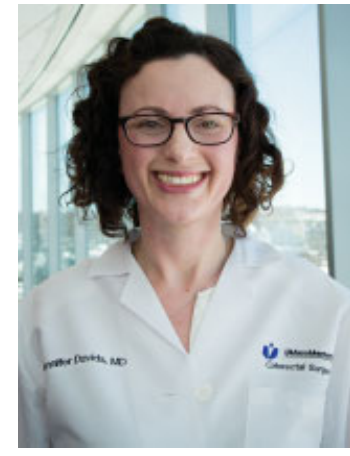

Jennifer S. Davids, MD, FACS, FASCRS to other opportunities.

While it is imperative that burnout and other threats to surgeon health are addressed on national, regional, and local levels, we must also educate ourselves and our colleagues. Numerous obstacles to reform exist on an organizational level, but change starts with each and every one of us.

I extend my sincere gratitude to Editor Dr. Scott Steele for giving me the opportunity to guest edit this volume. His enthusiasm and commitment to our specialty is infectious, and I am so "amped up" to have had the chance to work with him. Thank you to all the contributors who worked tirelessly to craft manuscripts that not only reflect current data but also include their invaluable personal experiences and perspectives.
Address for correspondence Jennifer S. Davids, MD, FACS FASCRS, Division of Colon and Rectal Surgery, UMass Memorial Medical Center, 67 Belmont St., Suite 201, Worcester, MA 01605 (e-mail: Jennifer.Davids@ umassmemorial.org).
Issue Theme Surgeon Health; Guest Editor: Jennifer S. Davids, MD, FACS, FASCRS
Copyright $\odot 2019$ by Thieme Medical Publishers, Inc., 333 Seventh Avenue, New York, NY 10001, USA. Tel: +1(212) 584-4662.
DOI https://doi.org/ 10.1055/s-0039-1692708. ISSN 1531-0043. 